\title{
Structure of the C. elegans Innexin-6 Gap Junction Channel
}

\author{
Atsunori Oshima ${ }^{1,2}$, Kazutoshi Tani $^{1}$ and Yoshinori Fujiyoshi ${ }^{1,2}$ \\ 1. Cellular and Structural Physiology Institute (CeSPI), Nagoya University, Furo-cho, Chikusa-ku, \\ Nagoya, Japan \\ 2. Department of Basic Medicinal Sciences, Graduate School of Pharmaceutical Sciences, Nagoya \\ University, Furo-cho, Chikusa-ku, Nagoya, Japan
}

Gap junction channels directly connect the cytoplasm of two adjacent cells, and play critical roles in many biologically important processes, including cardiac and nervous system development, fertility, immunity, and electrical synapse formation. Two protein families are involved in the formation of these channels, innexins for invertebrates and connexins for chordates, and there is no significant sequence similarity between the two. Connexins are assembled into a dodecameric channel, and the N-terminal pore funnel configuration has been reported for connexin-26 (Cx26) [1]. We have reported an electron crystallographic structure of the N-terminal deleted Caenorhabditis elegans innexin-6 (INX-6) showing a hexadecameric channel [2]. No high-resolution structure of innexin had yet been reported, however, and the cytoplasmic domains of the gap junction channel had not been visualized in any previous studies on gap junction structure.

Recently single-particle analysis cryo-electron microscopy (cryo-EM) has emerged as a powerful tool to determine protein structures at atomic or near atomic resolution without the need for crystallization. While it is essential to form perfectly thin ice layers for high resolution cryo-EM analysis, the presence of free detergent micelles in purified membrane protein solution sometimes make this process complicated. GraDeR is a method in which free detergent micelles are removed from solubilized membrane protein solution by glycerol gradient centrifugation [3, 4].

Here we report the cryo-EM structures of wild type INX-6 gap junction channels at atomic resolution [5]. As confirmed by negative stain EM, GraDeR removed most of free detergent micelles (Fig. 1). The INX-6 hemichannel and gap junction channel were reconstructed at $3.3 \AA$ resolution (Fig. 2(a)) and 3.6 $\AA$ resolution, respectively, allowing us to construct the first atomic model of a full gap junction channel (Fig. 2(b)). We found that the arrangements of transmembrane helices, $\beta$-hairpin and disulfide bonds in the two extracellular loops of the INX-6 monomeric structure are highly similar to those of Cx26, despite the lack of significant sequence similarity (Fig. 2(c)). The INX-6 gap junction channel comprises hexadecameric subunits but reveals an N-terminal pore funnel, consistent with $\mathrm{Cx} 26$. The helix-rich cytoplasmic loop and C-terminus are intercalated one-by-one through an octameric hemichannel, forming a dome-like entrance that interacts with $\mathrm{N}$-terminal loops in the pore (Fig. 2(d)).

These observations suggest that the INX-6 cytoplasmic domains are cooperatively associated with the $\mathrm{N}$-terminal funnel conformation, and an essential linkage of the N-terminal with channel activity is presumably preserved across gap junction families. The structural similarity between INX-6 and Cx26 suggests that these two families share a common primordial ancestor followed by long-term divergent evolution while maintaining a similar function. Finally, the GraDeR method $[3,4]$ we used for detergent removal could become an important tool for high-resolution analyses of membrane proteins based on single-particle cryo-electron microscopy [6]. 
References:

[1] S Maeda et al, Nature 458 (2009), p. 597.

[2] A Oshima et al, J. Mol. Biol. 428 (2016), p. 1227.

[3] F Hauer et al, Structure 23 (2015), p. 1769.

[4] F Hauer and C Gerle www.grader-protocol.com (2017).

[5] A Oshima, K Tani and Y Fujiyoshi. Nat. Commun. 7 (2016), p. 13681.

[6] This work was supported by Grants-in-Aid for Scientific Research (S), the Japan New Energy and Industrial Technology Development Organization (NEDO), the Japan Agency for Medical Research and Development (AMED) (Y.F.); Grants-in-Aid for Scientific Research (C) (K.T.); Grants-in-Aid for Young Scientists (B), Grants-in-Aid for Scientific Research (C) and the Platform for Drug Design, Discovery, and Development from MEXT, Japan (A.O.).
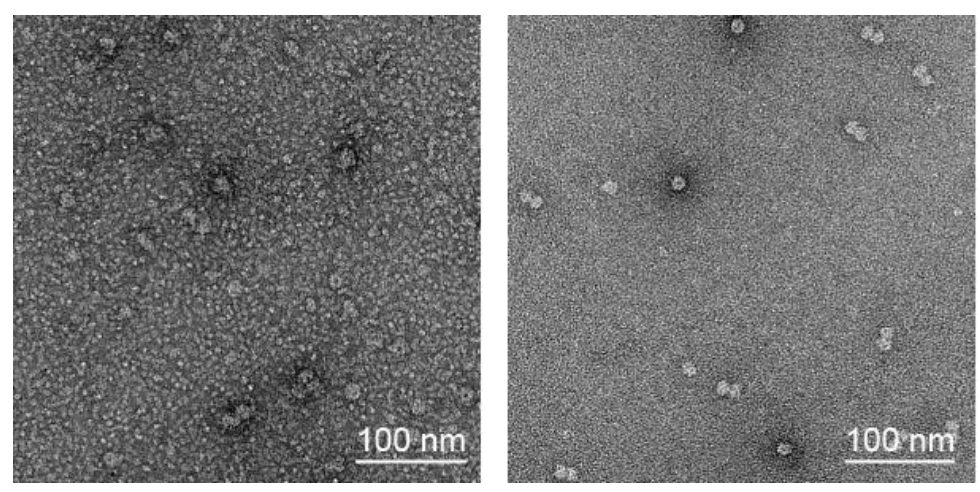

Figure 1. Negatively-stained electron micrographs of INX-6 channels before (left) and after (right) GraDeR [5].

(a)

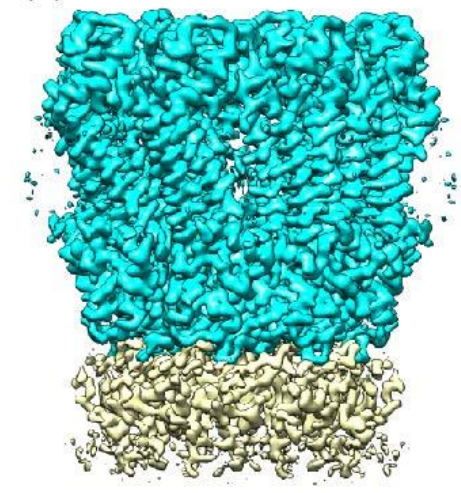

(b)

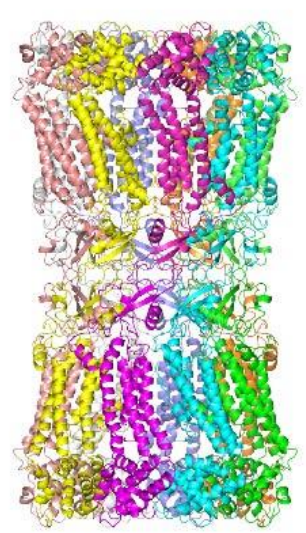

(c)

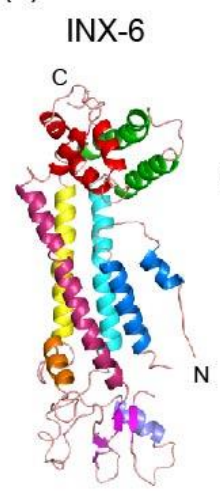

(d)

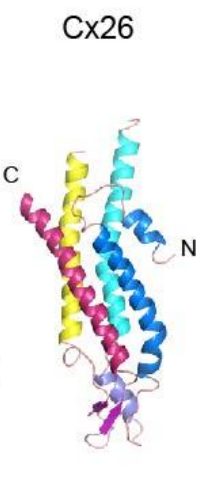

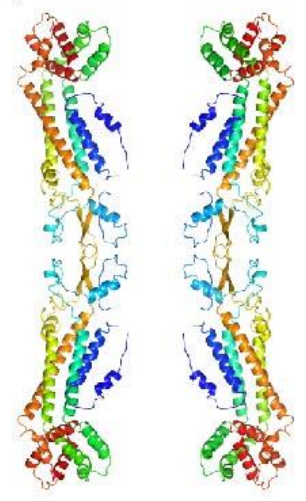

Figure 2. Cryo-EM structure and atomic model of INX-6 channel [5]. (a) Cryo-EM reconstruction of INX-6 hemichannel at 3.3A resolution. (b) Ribbon model of the full INX-6 gap junction channel. (c) The monomeric structures of INX-6 and Cx26 (pdb code: 2zw3) [1]. (d) The pore pathway shown by four INX-6 subunits. 